\title{
On the spectrum-generating superalgebras of the deformed one-dimensional quantum oscillators
}

\author{
N. Aizawa, $\quad$ I. E. Cunha ${ }^{\dagger}$ Z. Kuznetsova ${ }^{\ddagger}$ and F. Toppan ${ }^{\S}$
}

June 25, 2021

* Department of Physical Sciences, Graduate School of Science, Osaka Prefecture University, Nakamozu Campus, Sakai, Osaka 599-8531 Japan.

t§ CBPF, Rua Dr. Xavier Sigaud 150, Urca, cep 22290-180, Rio de Janeiro (RJ), Brazil.

$\ddagger$ UFABC, Av. dos Estados 5001, Bangu, cep 09210-580, Santo André (SP), Brazil.

\begin{abstract}
We investigate the dynamical symmetry superalgebras of the one-dimensional matrix superconformal quantum mechanics with inverse-square potential. They act as spectrumgenerating superalgebras for the systems with the addition of the de Alfaro-Fubini-Furlan oscillator term. The undeformed quantum oscillators are expressed by $2^{n} \times 2^{n}$ supermatrices; their corresponding spectrum-generating superalgebras are given by the $\operatorname{ssp}(2 n \mid 2)$ series. For $n=1$ the addition of a inverse-square potential does not break the $\operatorname{sep}(2 \mid 2)$ spectrum-generating superalgebra. For $n=2$ two cases of inverse-square potential deformations arise. The first one produces Klein deformed quantum oscillators; the corresponding spectrum-generating superalgebras are given by the $D(2,1 ; \alpha)$ class, with $\alpha$ determining the inverse-square potential coupling constants. The second $n=2$ case corresponds to deformed quantum oscillators of non-Klein type. In this case the $\operatorname{spp}(4 \mid 2)$ spectrum-generating superalgebra of the undeformed theory is broken to $\operatorname{osp}(2 \mid 2)$. The choice of the Hilbert spaces corresponding to the admissible range of the inverse-square potential coupling constants and the possible direct sum of lowest weight representations of the spectrum-generating superalgebras is presented.
\end{abstract}

Revised version: March 8, 2019

CBPF-NF-004/18

\footnotetext{
*E-mail: aizawa@p.s.osakafu-u.ac.jp

${ }^{\dagger}$ E-mail: ivanec@cbpf.br

${ }^{\ddagger}$ E-mail: zhanna.kuznetsova@ufabc.edu.br

${ }^{\S}$ E-mail: toppan@cbpf.br
} 


\section{Introduction and summary}

In this paper we present a systematic investigation of the one-dimensional matrix oscillators deformed by diagonal inverse-square potentials. We derive the general conditions for the existence of (one-dimensional, superconformal) spectrum-generating superalgebras. We give the most general solutions (up to similarity transformations and with at least $\mathcal{N}=2$ supersymmetries) for $2 \times 2$ and $4 \times 4$ supermatrices. For these cases we compute the admissible Hilbert spaces and prove that, depending on the range of the inverse-square potential coupling constants, the Hilbert space can be identified with a single lowest weight representation of the spectrumgenerating superalgebra or with a direct sum of its lowest weight representations. In the latter case the selection of the Hilbert space for the given model is not unique. This feature was already noted in [1, 2] for the purely bosonic case of ordinary (i.e., not matrix) inverse-square potential quantum mechanics [3] and de Alfaro-Fubini-Furlan oscillator [4]. In this work, among other results, we extend the [1, 2] analysis to the (super)matrix case.

The one-dimensional $2^{n} \times 2^{n}$ undeformed matrix oscillators possess $\operatorname{osp}(2 n \mid 2)$ spectrum generating superalgebras. For $n=1$ the addition of a inverse-square potential does not break the $\operatorname{osp}(2 \mid 2)$ spectrum-generating superalgebra. For $n=2$ two cases of inverse-square potential deformations arise. The first one produces Klein deformed quantum oscillators [5] (see also [6] and references therein for Klein oscillators); the corresponding spectrum-generating superalgebras are given by the $D(2,1 ; \alpha)$ class, with $\alpha$ determining the inverse-square potential coupling constants. The second $n=2$ case corresponds to deformed quantum oscillators of non-Klein type. In this case the $\operatorname{osp}(4 \mid 2)$ spectrum-generating superalgebra of the undeformed theory is broken to $\operatorname{osp}(2 \mid 2)$.

The topic under investigation is receiving considerable attention in the literature. Three frameworks are presently used for investigations. The most popular one consists in the quantization of (superconformal) worldline sigma-models; a second approach consists in analyzing symmetries (following, e.g., [7]) of partial differential equations which, for the present case, are time-dependent Schrödinger equations of matrix type; the third approach is the one here employed. The main motivations for investigating classical worldline superconformal sigma-models (and their quantization) come from the recognition that they underlie the dynamics of test particles in the proximity of the horizon of certain black holes (see [8]) and for their role in the $A d S_{2} / C F T_{1}$ correspondence [9, 10]. The worldline superconformal sigma-models (for a review see [11] and references therein) are obtained by imposing constraints to the supersymmetric sigma-models associated with one-dimensional supermultiplets [12, 13]. They can be derived by using superspace [11] or $D$-module representations of superconformal algebras [14, 15]. In [16], extending the construction pointed out in [17], it was shown that superconformal dynamical symmetries of worldline sigma-models are obtained from either parabolic or trigonometric $D$-module representations of the superconformal algebra. The first case corresponds to the classical version of the inverse-square potential, while the second case corresponds to the classical addition of the de Alfaro-Fubini-Furlan oscillator term. The quantization of the parabolic superconformal sigma-models has been performed in several papers (see, e.g., [18, 19] for the $D(2,1 ; \alpha)$ superconformal models). The quantization of trigonometric superconformal sigma-models has been done in fewer works (in [20, 21] for undeformed oscillators, while the first example of deformed oscillator was produced in [22]). The quantization of these systems is given in terms of quantum Noether charges which are expressed in the Heisenberg framework. We point out that the above papers lack the full analysis of the selection of the admissible Hilbert spaces as done in [1, 2] and the present work.

The approach based on the symmetry of a matrix partial differential equation was dis- 
cussed in [23] for a specific model. The deformed oscillator system described in Section 4 is the time-independent version of the time-dependent Schrödinger equation introduced in [23]. The anaysis of this model is made here more explicit in three points, namely the recognition that the $\operatorname{osp}(1 \mid 2) \subset \operatorname{osp}(2 \mid 2)$ subalgebra is sufficient to determine the spectrum of the theory, the selection of the admissible Hilbert spaces in the three different intervals $\left(\beta \leq-\frac{1}{2},-\frac{1}{2}<\beta<\frac{1}{2}, \beta \geq \frac{1}{2}\right)$ of the deformation parameter $\beta$ and, finally, the computation of the orthonormal eigenstates.

The model with $D(2,1 ; \alpha)$ spectrum-generating superalgebra discussed in Section $\mathbf{5}$ was first derived in [22]. We present here a more thorough analysis which includes the selection of the admissible Hilbert spaces for the three different intervals of the deformation parameter $\alpha$, as well as the recognition that the $\operatorname{osp}(2 \mid 2) \subset D(2,1 ; \alpha)$ subalgebra is sufficient to determine the spectrum of the theory. This extra analysis is made possible by the simplification which occurs in presenting the operators of the spectrum-generating superalgebra in a Schrödinger framework (therefore, with no time dependence) instead of the Heisenberg framework of [22].

The deformed oscillator (whose spectrum and orthonormal eigenstates have been computed) introduced in Section 6 is a genuine new model. It is the simplest example of a inverse-square deformed oscillator of non-Klein type. Its spectrum-generating superalgebra is $\operatorname{osp}(2 \mid 2)$. The model depends on a real deformation parameter $\nu$. The Hilbert space exists (and is unique, being given by a lowest weight representation of $\operatorname{osp}(2 \mid 2))$ for $\nu \neq 0$. The vacuum is unique, while all excited states are doubly degenerate, so that the semiinfinite $(1,2,2,2, \ldots)$ tower of states is produced. Unlike the Klein-deformed matrix oscillators (38 61), the non-Klein deformed matrix oscillator 80 is not obtained as a continuous deformation of the undeformed oscillator.

The scheme of the paper is as follows. In Section $\mathbf{2}$ we derive the spectrum-generating superalgebras of the undeformed one-dimensional matrix oscillators. In Section $\mathbf{3}$ we discuss their deformations via the introduction of diagonal inverse-square potentials. The $n=1$ example of deformed oscillators is presented in Section 4. In Section 5 the analysis is extended to the Klein deformations of the $n=2$ oscillators. In Section 6 we produce the results for the nonKlein deformation of the $n=2$ matrix oscillators. Some comments about the present knowledge of the existing deformations for $n \geq 3$ are given in Section 7. In the Conclusions we mention open problems for further investigations. The paper is complemented by three Appendices. In Appendix A we discuss the relevant features of the subclass of finite Lie superalgebras which are one-dimensional superconformal. In Appendix $\mathbf{B}$ we present the basic properties of the exceptional class of $D(2,1 ; \alpha)$ superalgebras. Finally, in Appendix $\mathbf{C}$, we discuss the selection of the admissible Hilbert spaces for the de Alfaro-Fubini-Furlan deformed oscillators.

\section{The undeformed one-dimensional quantum oscillators and their $o s p(2 n \mid 2)$ spectrum-generating superalgebras}

As recalled in [24], the (96) superalgebra (see Appendix A) of the supersymmetric quantum mechanics can be constructed by Hermitian matrix differential operators $Q_{I}, H$ acting on a supermultiplet of real-valued fields. On the other hand the introduction of a dynamical symmetry realized by Hermitian operators closing a superconformal algebra requires a complex structure. The reason is the presence of non-vanishing commutators ( $\operatorname{such}$ as $\left[Q_{I}, K\right]=i \widetilde{Q}_{I}$ ); they imply that the imaginary unit has to be introduced in order to have Hermitian operators on the right hand side. Therefore, without loss of generality, we can investigate superconformal dynamical symmetries (and spectrum-generating superalgebras) acting on supermultiplets of complex fields. 
The one-dimensional $2^{n} \times 2^{n}$ free matrix Hamiltonian $H$ is given by

$$
H=-\frac{1}{2} \partial_{x}^{2} \cdot \mathbb{I}_{2^{n}}
$$

(here and in the following $\mathbb{I}_{k}$ denotes the $k \times k$ identity matrix).

For any positive integer $n \in \mathbb{N}, H$ possesses $2 n$ distinct Hermitian, fermionic (i.e. blockantidiagonal) first-order matrix differential operators $Q_{I}$ as its square roots. The $Q_{I}$ operators close the $\mathcal{N}$-extended superalgebra (96) with

$$
\mathcal{N}=2 n
$$

The above relation between $\mathcal{N}$ and $2 n$ is based on the constructions reported in [25, 26] for complex-valued Clifford algebras. There are $2 n$ block antidiagonal complex matrices $\gamma_{I}, I=$ $1,2, \ldots, 2 n$, satisfying the relations

$$
\gamma_{I} \gamma_{J}+\gamma_{J} \gamma_{I}=2 \delta_{I J} \cdot \mathbb{I}_{2^{n}}
$$

The extra block-diagonal matrix $F$,

$$
F=\left(\begin{array}{cc}
\mathbb{I}_{2^{n-1}} & 0 \\
0 & -\mathbb{I}_{2^{n-1}}
\end{array}\right)
$$

satisfies the anticommutation relations

$$
F \gamma_{I}+\gamma_{I} F=0, \quad \forall I=1,2, \ldots, 2 n
$$

$F$ is called the fermion parity operator. Its eigenvectors with $+1(-1)$ eigenvalue are the even, also called bosonic (odd, also called fermionic), states.

We can set

$$
Q_{I}=\frac{i}{\sqrt{2}} \gamma_{I} \partial_{x}
$$

so that $(96)$ reads as

$$
\left\{Q_{I}, Q_{J}\right\}=2 \delta_{I J} H, \quad\left[H, Q_{I}\right]=0, \quad \text { for } \quad I, J=1, \ldots, 2 n .
$$

The conformal counterpart of the Hamiltonian $H$ is the oscillator $K$ which can be assumed to be proportional to the identity matrix. Therefore

$$
K=\frac{1}{2} x^{2} \cdot \mathbb{I}_{2^{n}}
$$

The conformal counterparts of the $Q_{I}$ operators are the Hermitian operators $\widetilde{Q}_{I}$, introduced through

$$
\left[Q_{I}, K\right]=i \widetilde{Q}_{I} \rightarrow \widetilde{Q}_{I}=\frac{1}{\sqrt{2}} x \cdot \gamma_{I}
$$

The dilatation operator $D$ and the $R$-symmetry operators $\Sigma_{I J}=-\Sigma_{J I}$ are introduced from the anticommutators

$$
\left\{Q_{I}, \widetilde{Q}_{J}\right\}=-2 \delta_{I J} D+\Sigma_{I J}
$$


We have

$$
\begin{aligned}
D & =-\frac{i}{2}\left(x \partial_{x}+\frac{1}{2}\right) \cdot \mathbb{I}_{2^{n}}, \\
\Sigma_{I J} & =\frac{i}{2} \gamma_{I} \gamma_{J} .
\end{aligned}
$$

For any positive integer $n$ the set of Hermitian operators $D, H, K, Q_{I}, \widetilde{Q}_{I}, \Sigma_{I J}$ close the $D(n, 1) \sim$ $o s p(2 n \mid 2)$ superalgebra. The $4 n$ generators $Q_{I}, \widetilde{Q}_{I}$ are odd. The $n(2 n-1)+3$ even generators $H, D, K, \Sigma_{I J}$ produce the $s l(2) \oplus s o(2 n)$ subalgebra. The superalgebra $\operatorname{osp}(2 n \mid 2)$ belongs to the class of one-dimensional superconformal algebras discussed in Appendix A (the $s o(2 n)$ subalgebra is the $R$-symmetry).

We present, for completeness, the non-vanishing (anti)commutators of $\operatorname{ssp}(2 n \mid 2)$. In order to write them in more compact form we introduce the generators $E^{+}=H, E^{-}=K, Q_{I}^{+}=Q_{I}$, $Q_{I}^{-}=\widetilde{Q}_{I}$. We have

$$
\begin{aligned}
& {\left[D, E^{ \pm}\right]= \pm i E^{ \pm}, \quad\left[D, Q_{I}^{ \pm}\right]= \pm \frac{i}{2} Q_{I}^{ \pm},} \\
& {\left[E^{+}, E^{-}\right]=-2 i D, \quad\left\{Q_{I}^{+}, Q_{I}^{-}\right\}=-2 \delta_{I J} D+\Sigma_{I J},} \\
& {\left[E^{ \pm}, Q_{I}^{\mp}\right]= \pm i Q_{I}^{ \pm}, \quad\left\{Q_{I}^{ \pm}, Q_{J}^{ \pm}\right\}=2 \delta_{I J} E^{ \pm},} \\
& {\left[\Sigma_{I J}, \Sigma_{I L}\right]=-i \Sigma_{J L}, \quad\left[\Sigma_{I J}, Q_{K}^{ \pm}\right]=-i \delta_{I K} Q_{J}^{ \pm}+i \delta_{J K} Q_{I}^{ \pm} .}
\end{aligned}
$$

The Hamiltonian $H_{o s c}$ of the (undeformed) matrix oscillator is the sum of $H$ and $K$ :

$$
H_{\text {osc }}=H+K=\frac{1}{2}\left(-\partial_{x}^{2}+x^{2}\right) \cdot \mathbb{I}_{2^{n}} .
$$

The superalgebra $\operatorname{osp}(2 n \mid 2)$ is the spectrum-generating superalgebra for $H_{\text {osc }}$. A linear combination of the odd generators produce $2 n$ pairs of $a_{I}^{\dagger}, a_{I}$ creation/annihilation operators satisfying $2 n$ independent Heisenberg algebras defined by their commutators.

We can set

$$
a_{I}=Q_{I}+i \widetilde{Q}_{I}=\frac{i}{\sqrt{2}} \gamma_{I}\left(\partial_{x}+x\right), \quad a_{I}^{\dagger}=Q_{I}-i \widetilde{Q}_{I}=\frac{i}{\sqrt{2}} \gamma_{I}\left(\partial_{x}-x\right) .
$$

In terms of anticommutators we have (no summation over the repeated indices is understood)

$$
H_{o s c}=\frac{1}{2}\left\{a_{I}, a_{I}^{\dagger}\right\} .
$$

$a_{I}^{\dagger}\left(a_{I}\right)$ are creation (annihilation) operators due to the commutators

$$
\left[H_{o s c}, a_{I}^{\dagger}\right]=a_{I}^{\dagger}, \quad\left[H_{o s c}, a_{I}\right]=-a_{I} .
$$

For every $I$, the Heisenberg algebras are recovered from

$$
\left[a_{I}, a_{I}^{\dagger}\right]=\mathbb{I}_{2^{n}}
$$

The annihilation operators $a_{I}$ allow to define the $2^{n}$ degenerate ground states $|0\rangle_{I}$ of $H_{\text {osc }}$ as the lowest weight vectors satisfying, for each $I$,

$$
a_{I}|0\rangle_{I}=0, \quad H_{\text {osc }}|0\rangle_{I}=\frac{1}{2}|0\rangle_{I} .
$$

Half of the degenerate ground states are bosonic and half of them are fermionic. The Hilbert space of the undeformed matrix oscillator is a $2^{n}$-ple of $\mathcal{L}^{2}(\mathbb{R})$ square integrable functions on the line. 


\section{The inverse-square potential in matrix quantum Hamiltonians}

The addition to the free Hamiltonian $H$ in (1) of a inverse-square potential $\frac{1}{x^{2}} V$, where $V=$ $\operatorname{diag}\left(v_{1}, v_{2}, \ldots v_{2^{n}}\right)$ is a $2^{n} \times 2^{n}$ constant diagonal matrix, is such to preserve the scaling property of $H$. Indeed, if we set the scaling dimension of the space coordinate to be $[x]=-1$, then

$$
[H]=\left[H+\frac{1}{x^{2}} V\right]=2 .
$$

In this paper we address the question of the constraints to be imposed on the inverse-square potential coupling constants $v_{i}$ 's entering the diagonal matrix $V$ in order to get a one-dimensional superconformal Lie algebra as a dynamical symmetry of the inverse-square deformed Hamiltonian $H_{\text {def }}$, defined as

$$
H_{\text {def }}=H+\frac{1}{x^{2}} V
$$

By construction the associated inverse-square deformed oscillator $H_{\text {osc }}+\frac{1}{x^{2}} V$, with $H_{\text {osc }}$ given in (13), possesses the obtained one-dimensional superconformal Lie algebra as a spectrumgenerating superalgebra.

Obviously the $\operatorname{ssp}(2 n \mid 2)$ dynamical symmetry of the free Hamiltonian is in general no longer a dynamical symmetry of the $\frac{1}{x^{2}} V$ inverse-square deformed Hamiltonian. It is worth noticing, on the other hand, that the dynamical symmetry of the inverse-square deformed Hamiltonian is not necessarily a subalgebra of $\operatorname{osp}(2 n \mid 2)$, as one could naïvely expect. In some cases (discussed in the following in Sections $\mathbf{5}$ and $\mathbf{7})$ it corresponds to a deformation of $\operatorname{ssp}(2 n \mid 2)$.

The deformed supersymmetry operators have to be expressed as

$$
Q_{I}^{\text {def }}=\frac{i}{\sqrt{2}}\left(\gamma_{I} \partial_{x}-i \frac{M_{I}}{x}\right)
$$

where the $M_{I}$ 's should be block-antidiagonal, constant matrices satisfying the hermiticity condition $M_{I}^{\dagger}=M_{I}$.

The closure of the 96 superalgebra requires the following equations to be satisfied for $I \neq J$

$$
\begin{aligned}
\left\{\gamma_{I}, M_{J}\right\}+\left\{\gamma_{J}, M_{I}\right\} & =0 \\
\left\{M_{I}, M_{J}\right\}-i \gamma_{I} M_{J}-i \gamma_{J} M_{I} & =0 .
\end{aligned}
$$

At $I=J$ the potential of the inverse-square potential deformed Hamiltonian $H_{\text {def }}$ should be given by $\frac{1}{x^{2}} V$ where, for any $I$, we get

$$
V=\frac{1}{2}\left(M_{I}^{2}-i \gamma_{I} M_{I}\right)
$$

The (8) oscillator operator $K$ remains undeformed; it follows that the dilatation operator $D$ and the fermionic operators $\widetilde{Q}_{I}$ are also unchanged.

In order to recover the dilatation operator $D$ from the anticommutator $\left\{Q_{I}^{\text {def }}, \widetilde{Q}_{I}\right\}$, for any $I$ the condition

$$
\left\{M_{I}, \gamma_{I}\right\}=0
$$

has to be fulfilled.

The anticommutators $\left\{Q_{I}^{\text {def }}, \widetilde{Q}_{J}\right\}$ for $I \neq J$ give the constant operators

$$
\Sigma_{I J}^{\text {def }}=\frac{1}{2}\left(\frac{i}{2}\left[\gamma_{I}, \gamma_{J}\right]+\left\{M_{I}, \gamma_{J}\right\}\right)
$$


Since the first relation in 22 is assumed to be satisfied, then $\Sigma_{I J}^{d e f}=-\Sigma_{J I}^{d e f}$.

The closure of a superconformal algebra is obtained provided that the $\Sigma_{I J}^{d e f}$ 's close the $R$ symmetry subalgebra and that the fermionic operators $Q_{I}^{\text {def }}, \widetilde{Q}_{I}$ belong to $R$-symmetry representation multiplets.

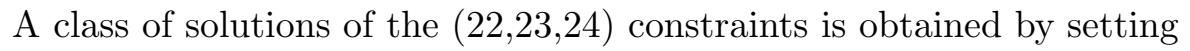

$$
M_{I}=i \beta \gamma_{I} F
$$

where $\beta$ is an arbitrary real number and $F$ is the fermion parity operator introduced in (4). The (26) solution fails, however, to produce a superconformal algebra for $n \geq 3$.

The $\Sigma_{I J}^{\text {def }}$ operators from 25 , under 26 deformation, read

$$
\Sigma_{I J}^{d e f}=\frac{i}{2} \gamma_{I} \gamma_{J}(1-2 \beta F)
$$

It is immediate to check that, for $n=1$, the introduction of the 26$)$ deformation does not spoil the $\operatorname{osp}(2 \mid 2)$ dynamical symmetry of the free system.

For $n=2$ the closure of a superconformal algebra as a dynamical symmetry is guaranteed by the fact that $F$ is expressed by the product $F=\gamma_{1} \gamma_{2} \gamma_{3} \gamma_{4}$. This relation implies that the commutators $\left[\Sigma_{I J}^{\text {def }}, \widetilde{Q}_{K}\right]$ close on the $\widetilde{Q}_{L}$ generators on the right hand side. A similar property holds for the $Q_{K}$ generators.

The models based on the (26) deformation for $n=1$ and $n=2$ are explicity discussed in Section 4 and, respectively, Section 5.

The (26) deformation implies that the deformed creation and annihilation operators satisfy a Klein-deformed Heisenberg algebra. We recall (see [6]) that the Klein-deformed Heisenberg algebra is realized by a pair of Hermitian conjugated operators $a_{K l}, a_{K l}^{\dagger}$ satisfying the relations

$$
\begin{aligned}
{\left[a_{K l}, a_{K l}^{\dagger}\right] } & =\mathbb{I}+\nu \bar{K}, \\
\left\{a_{K l}, \bar{K}\right\} & =\left\{a_{K l}^{\dagger}, \bar{K}\right\}=0, \\
\bar{K}^{2} & =\mathbb{I},
\end{aligned}
$$

for some given real number $\nu$. The operator $\bar{K}$, which is a square root of the Identity, is known as "Klein operator".

In term of the (26) deformation we can set

$$
\begin{aligned}
& a_{K l, I}=Q_{I}^{\text {def }}+i \widetilde{Q}_{I}=\frac{i}{\sqrt{2}} \gamma_{I}\left(\partial_{x}+x+\frac{\beta F}{x}\right), \\
& a_{K l, I}^{\dagger}=Q_{I}^{\text {def }}-i \widetilde{Q}_{I}=\frac{i}{\sqrt{2}} \gamma_{I}\left(\partial_{x}-x+\frac{\beta F}{x}\right) .
\end{aligned}
$$

It follows

$$
\left[a_{K l, I}, a_{K l, I}^{\dagger}\right]=\mathbb{I}-2 \beta F, \quad\left\{a_{K l, I}, F\right\}=\left\{a_{K l, I}^{\dagger}, F\right\}=0 .
$$

For any $I$ the deformed creation/annihilation operators (29) define a 28) Klein-deformed Heisenberg algebra with $\nu=-2 \beta$ and fermion parity operator $F$ as Klein operator.

Furthermore, we get the deformed oscillator Hamiltonian $H_{o s c}^{K l}$ from the anticommutators

$$
\frac{1}{2}\left\{a_{K l, I}, a_{K l, I}^{\dagger}\right\}=H_{o s c}^{K l}=H_{d e f}+K=\frac{1}{2}\left(-\partial_{x}^{2}+x^{2}+\frac{\beta^{2}+\beta F}{x^{2}}\right) \mathbb{I} .
$$


The Klein-deformed oscillators are creation/annihilation operators since

$$
\left[H_{o s c}^{K l}, a_{K l, I}\right]=-a_{K L, I}, \quad\left[H_{o s c}^{K l}, a_{K l, I}^{\dagger}\right]=a_{K L, I}^{\dagger} .
$$

We close this Section by pointing out that the constraints 222324 admit more general solutions, different from the ones given by (26). These solutions can also induce superconformal algebras as dynamical symmetries. One of such examples, leading to deformed creation/annihilation oscillators which do not satisfy the Klein condition, is presented in Section 6.

\section{The $n=1$ case with Klein deformed oscillators and $\operatorname{osp}(2 \mid 2)$ spectrum-generating superalgebra}

In this Section we present the $n=1$ Klein deformed oscillator. We show that its spectrumgenerating superalgebra is $\operatorname{osp}(2 \mid 2)$, like the undeformed case. The construction of the admissible Hilbert spaces is given at the end.

For $n=1$ the formulas of the operators given in Section 3 are specialized in terms of the three Pauli matrices $\sigma_{i}, i=1,2,3$, given by

$$
\sigma_{1}=\left(\begin{array}{cc}
0 & 1 \\
1 & 0
\end{array}\right), \quad \sigma_{2}=\left(\begin{array}{cc}
0 & -i \\
i & 0
\end{array}\right), \quad \sigma_{3}=\left(\begin{array}{cc}
1 & 0 \\
0 & -1
\end{array}\right) .
$$

For any real value of the parameter $\beta$ the four even operators $H, D, K, J$ and the four odd operators $Q_{1}, Q_{2}, \widetilde{Q}_{1}, \widetilde{Q}_{2}$ close the $\operatorname{osp}(2 \mid 2)$ superalgebra. Their respective expressions are

$$
\begin{aligned}
H & =\frac{1}{2}\left(-\partial_{x}^{2}+\frac{\beta^{2}+\beta \sigma_{3}}{x^{2}}\right) \cdot \mathbb{I}_{2} \\
D & =-\frac{i}{2}\left(x \partial_{x}+\frac{1}{2}\right) \cdot \mathbb{I}_{2} \\
K & =\frac{1}{2} x^{2} \cdot \mathbb{I}_{2} \\
J & =-\frac{1}{2} \sigma_{3}+\beta \mathbb{I}_{2} \\
Q_{I} & =\frac{i}{\sqrt{2}} \sigma_{I} \cdot\left(\partial_{x}+\frac{\beta \sigma_{3}}{x}\right) \\
\widetilde{Q}_{I} & =\frac{1}{\sqrt{2}} \sigma_{I} \cdot x
\end{aligned}
$$

where $I=1,2$.

Their non-vanishing (anti)commutators are given in Appendix A, formula (98).

One should note that the closure of the $\operatorname{ssp}(2 \mid 2)$ superalgebra is not affected by the presence of the non-vanishing real parameter $\beta$. The reality condition on $\beta$ is imposed to guarantee the hermiticity property of the (34) operators.

The Klein-deformed oscillators are introduced through the positions

$$
a_{I}=Q_{I}+i \widetilde{Q}_{I}, \quad a_{I}^{\dagger}=Q_{I}-i \widetilde{Q}_{I} .
$$

Therefore we obtain, for $I=1,2$,

$$
\begin{aligned}
& a_{I}=\frac{i}{\sqrt{2}} \sigma_{I} \cdot\left(\partial_{x}+\frac{\beta \sigma_{3}}{x}+x\right), \\
& a_{I}^{\dagger}=\frac{i}{\sqrt{2}} \sigma_{I} \cdot\left(\partial_{x}+\frac{\beta \sigma_{3}}{x}-x\right) .
\end{aligned}
$$


At a given $I=1,2$, the commutator is

$$
\left[a_{I}, a_{I}^{\dagger}\right]=\mathbb{I}_{2}-2 \beta \sigma_{3},
$$

while the Hamiltonian $H_{o s c}$ of the deformed oscillator is

$$
H_{o s c}=\frac{1}{2}\left\{a_{I}, a_{I}^{\dagger}\right\}=H+K=\frac{1}{2}\left(-\partial_{x}^{2}+x^{2}+\frac{\beta^{2}+\beta \sigma_{3}}{x^{2}}\right) \cdot \mathbb{I}_{2}
$$

The condition

$$
a_{I}|\lambda\rangle=0
$$

defines a lowest weight vector. For any real $\beta$ there are two such lowest weight vectors, one bosonic $\left(\left|\lambda_{\text {Bos }}\right\rangle\right.$, such that $\left.\sigma_{3}\left|\lambda_{\text {Bos }}\right\rangle=\left|\lambda_{\text {Bos }}\right\rangle\right)$ and one fermionic $\left(\left|\lambda_{F e r}\right\rangle\right.$, such that $\sigma_{3}\left|\lambda_{F e r}\right\rangle=$ $\left.-\left|\lambda_{\text {Fer }}\right\rangle\right)$.

We have that

$$
\left|\lambda_{\text {Bos }}\right\rangle \propto\left(\begin{array}{c}
x^{-\beta} e^{-\frac{1}{2} x^{2}} \\
0
\end{array}\right), \quad\left|\lambda_{F e r}\right\rangle \propto\left(\begin{array}{c}
0 \\
x^{\beta} e^{-\frac{1}{2} x^{2}}
\end{array}\right) .
$$

The annihilation operator $a_{2}$ defines, up to a phase, the same lowest weight vectors as $a_{1}$. This property remains true for the excited states: $\left(a_{2}^{\dagger}\right)^{n}\left|\lambda_{B o s}\right\rangle\left(\left(a_{2}^{\dagger}\right)^{n}\left|\lambda_{\text {Fer }}\right\rangle\right)$ differs from $\left(a_{1}^{\dagger}\right)^{n}\left|\lambda_{\text {Bos }}\right\rangle$ $\left(\left(a_{1}^{\dagger}\right)^{n}\left|\lambda_{\text {Fer }}\right\rangle\right)$ by a phase. It turns out that, as a spectrum-generating superalgebra of the $H_{\text {osc }}$ (38) Hamiltonian, $\operatorname{osp}(2 \mid 2)$ is redundant. The spectrum of the theory can be recovered from each one of the two copies of the $\operatorname{osp}(1 \mid 2) \subset \operatorname{osp}(2 \mid 2)$ subalgebras, either the one given by the generators $H, D, K, Q_{1}, \widetilde{Q}_{1}$, or the one given by the generators $H, D, K, Q_{2}, \widetilde{Q}_{2}$.

This important point deserves to be duly emphasized. We therefore present the

Remark: the spectrum-generating superalgebra osp $(2 \mid 2)$ is redundant to produce the spectrum of the theory since the ray vectors of a Hilbert space are determined by the osp $(1 \mid 2)$ spectrumgenerating subalgebra.

Repeating the analysis discussed in Appendix $\mathbf{C}$ to the present case, we easily conclude that the lowest weight representation induced by $\left|\lambda_{B o s}\right\rangle$ defines a normed Hilbert space given by a pair of $\mathcal{L}^{2}(\mathbb{R})$ square integrable functions on the real line, provided that the normalization condition $-2 \beta>-1$ is satisfied. Similarly, $\left|\lambda_{F e r}\right\rangle$ defines a Hilbert space provided that the condition $2 \beta>-1$ is satisfied.

We arrive at the following selection of admissible Hilbert spaces for the model:

i) in the range $\beta \leq-\frac{1}{2}$ the only admissible Hilbert space corresponds to a single lowest weight representation, with $\left|\lambda_{B o s}\right\rangle$ as ground state. Its vacuum energy $E_{B o s}(\beta)$ is given by $E_{\text {Bos }}(\beta)=\frac{1}{2}-\beta$;

ii) in the range $\beta \geq \frac{1}{2}$ the only admissible Hilbert space corresponds to a single lowest weight representation, with $\left|\lambda_{F e r}\right\rangle$ as ground state. Its vacuum energy $E_{F e r}(\beta)$ is given by $E_{F e r}(\beta)=\frac{1}{2}+\beta$;

iii) in the intermediate range $-\frac{1}{2}<\beta<\frac{1}{2}$ different choices of Hilbert space are admissible. Either

iiia) one can select as Hilbert space a single lowest weight representation (the lowest weight vector being either $\left|\lambda_{B o s}\right\rangle$ or $\left.\left|\lambda_{F e r}\right\rangle\right)$. Alternatively, 
iiib) the Hilbert space can be selected to be the direct sum of the two lowest weight representations. The energy difference $\Delta(\beta)=E_{B o s}(\beta)-E_{F e r}(\beta)$ of the two ground states is $\Delta(\beta)=-2 \beta$. Therefore, $\mid \lambda_{B o s}>$ is the vacuum state for $0<\beta<\frac{1}{2}$, while $\left|\lambda_{F e r}\right\rangle$ is the vacuum state for $-\frac{1}{2}<\beta<0$. A degenerate ground state is recovered for the $\beta=0$ undeformed oscillator.

We conclude this Section by pointing out that, without loss of generality, one can restrict the real parameter $\beta$ to belong to a half line (either $\beta \geq 0$ or $\beta \leq 0$ ). The reason for that is the existence of a similarity transformation, induced by the Pauli matrix $\sigma_{1}$, which allows to exchange bosonic and fermionic states. Under this similarity transformation any operator $g$ entering (34) is mapped into

$$
g^{\prime}=\sigma_{1} g \sigma_{1}
$$

Let us stress the $\beta$-dependence of $H$ entering (34) by denoting it as " $H(\beta)$ ". We obtain, in particular, that the following relation is satisfied

$$
H^{\prime}(\beta)=\sigma_{1} H(\beta) \sigma_{1}=H(-\beta)
$$

In the range $0<\beta<\frac{1}{2}$ the vacuum state is $\left|\lambda_{B o s}\right\rangle$. In terms of the iiib) option for the Hilbert space, the spectrum is given by

$$
E_{\epsilon, n}=\frac{1}{2}-\epsilon \beta+n,
$$

where $n \in \mathbb{N}_{0}$ and $\epsilon= \pm 1$. The vacuum energy corresponds to $\epsilon=1, n=0$.

Each energy level $E_{\epsilon, n}$ is not degenerate. The parity $P_{\epsilon, n}$ (even or odd) of the corresponding eigenfunctions, given by the \pm 1 eigenvalues of the fermion parity operator $\sigma_{3}$, is given by

$$
P_{\epsilon, n}=\epsilon(-1)^{n} \text {. }
$$

We compute now the orthonormality conditions for the corresponding eigenfunctions in the range $0<\beta<\frac{1}{2}$ (the orthonormality conditions for the Klein-deformed operators were presented in [6] and references therein). Let us denote as $|0\rangle_{\epsilon}$ the bosonic $(\epsilon=1)$ and the fermionic $(\epsilon=-1)$ normalized lowest weight states, so that ${ }_{\epsilon}\langle 0 \mid 0\rangle_{\epsilon}=1$. We determine $N_{ \pm 1}$ so that

$$
|0\rangle_{1}=N_{1}\left(\begin{array}{c}
x^{-\beta} e^{-\frac{1}{2} x^{2}} \\
0
\end{array}\right), \quad|0\rangle_{-1}=N_{-1}\left(\begin{array}{c}
0 \\
x^{\beta} e^{-\frac{1}{2} x^{2}}
\end{array}\right) .
$$

They are determined by the conditions, see [24],

$$
\left|N_{\epsilon}\right|^{2} \int_{-\infty}^{+\infty} d x\left|x^{-2 \epsilon \beta} e^{-x^{2}}\right|=1
$$

We express the line integral in terms of the Gamma function. At first we separate the line integral into two integrals: $\int_{-\infty}^{+\infty}=\int_{-\infty}^{0}+\int_{0}^{+\infty}$. By changing the integration variable $(x \mapsto-x)$ in the first integral on the right hand side we are able to write $\int_{-\infty}^{+\infty} d x\left|x^{-2 \epsilon \beta} e^{-x^{2}}\right|=(1+$ $\left.\left|(-1)^{-2 \epsilon \beta}\right|\right) \int_{0}^{+\infty} d x x^{-2 \epsilon \beta} e^{-x^{2}}=2 \int_{0}^{+\infty} d x x^{-2 \epsilon \beta} e^{-x^{2}}$. With the further change of the integration variable by setting $t=x^{2}$ we obtain $2 \int_{0}^{+\infty} d x x^{-2 \epsilon \beta} e^{-x^{2}}=\int_{0}^{+\infty} d t t^{-\epsilon \beta-\frac{1}{2}} e^{-t}=\Gamma\left(-\epsilon \beta+\frac{1}{2}\right)$. The normalization factors $N_{\epsilon}$ can therefore be expressed as

$$
N_{\epsilon}=\frac{1}{\sqrt{\Gamma\left(-\epsilon \beta+\frac{1}{2}\right)}} .
$$


The unnormalized excited states $|\bar{n}\rangle_{\epsilon}$ are introduced through the position

$$
|\bar{n}\rangle_{\epsilon}=\left(a^{\dagger}\right)^{n}|0\rangle_{\epsilon} .
$$

In the above formula, due to the previous remark on the redundancy of the $\operatorname{ssp}(2 \mid 2)$ superalgebra, the creation operator $a^{\dagger}$ can denote either $a_{1}^{\dagger}$ or $a_{2}^{\dagger}$. We denote with $a$ its corresponding annihilation operator satisfying (37). By exploiting the Klein-deformed commutator (37) and taking into account that $a|0\rangle_{\epsilon}=0$, we easily obtain the formulas

$$
a|\bar{n}\rangle_{\epsilon}=Z_{n}|\overline{n-1}\rangle_{\epsilon}, \quad \text { with } \quad Z_{2 k}=2 k, \quad Z_{2 k+1}=2 k+1-2 \epsilon \beta, \quad k \in \mathbb{N}_{0}
$$

(we set, for consistency, $|\overline{0}\rangle_{\epsilon}=|0\rangle_{\epsilon}$ ).

Let us introduce the normalization coefficients $M_{n, \epsilon}$ through the position

$$
M_{n, \epsilon}=\epsilon^{\langle\bar{n} \mid \bar{n}\rangle_{\epsilon}} .
$$

The equality ${ }_{\epsilon}\langle\overline{n+1} \mid \overline{n+1}\rangle_{\epsilon}={ }_{\epsilon}\left\langle\bar{n}\left|a a^{\dagger}\right| \bar{n}\right\rangle_{\epsilon}={ }_{\epsilon}\left\langle\bar{n}\left|\left(a a^{\dagger}-a^{\dagger} a+a^{\dagger} a\right)\right| \bar{n}\right\rangle_{\epsilon}$ implies the following recursive relation for $M_{n, \epsilon}$ :

$$
M_{n+1, \epsilon}=\left(1+2 \epsilon \beta(-1)^{n+1}\right) M_{n, \epsilon}+Z_{n}^{2} M_{n-1, \epsilon} .
$$

The first few terms are given by

$$
\begin{aligned}
& M_{0, \epsilon}=1, \\
& M_{1, \epsilon}=(1-2 \epsilon \beta), \\
& M_{2, \epsilon}=2(1-2 \epsilon \beta), \\
& M_{3, \epsilon}=(1-2 \epsilon \beta)(6-4 \epsilon \beta) .
\end{aligned}
$$

It is easily shown that the normalization of the undeformed oscillator is recovered in the limit $\beta \rightarrow 0$, since $M_{n, \epsilon} \rightarrow n$ !.

The orthornormal eigenstates, denoted as $|n\rangle_{\epsilon}$, are given by

$$
|n\rangle_{\epsilon}=\frac{1}{\sqrt{M_{n, \epsilon}}}|\bar{n}\rangle_{\epsilon} .
$$

The normalization condition can be defined in closed form in terms of the Pochhammer symbol, introduced through the position

$$
\begin{aligned}
& (x)_{n}=\frac{\Gamma(x+n)}{\Gamma(x)}=x(x+1)(x+2) \cdots(x+n-1), \quad n>0, \\
& (x)_{0}=1 .
\end{aligned}
$$

The repeated use of 490 implies

$$
\begin{aligned}
a_{I}^{2 k}|2 k\rangle_{\epsilon} & =2 k(2 k-1-2 \epsilon \beta)(2 k-2)(2 k-3-2 \epsilon \beta) \cdots 2(1-2 \epsilon \beta)|0\rangle_{\epsilon}= \\
& =(2 k) ! ! \frac{(-2 \epsilon \beta)_{2 k}}{(2 k-2-2 \epsilon \beta)(2 k-4-2 \epsilon \beta)(2-2 \epsilon \beta)(-2 \epsilon \beta)}|0\rangle_{\epsilon}= \\
& =\frac{(2 k) ! !}{2^{k}} \frac{(-2 \epsilon \beta)_{2 k}}{(-\epsilon \beta)_{k}}|0\rangle_{\epsilon}=\frac{k !(-2 \epsilon \beta)_{2 k}}{(-\epsilon \beta)_{k}}|0\rangle_{\epsilon} .
\end{aligned}
$$

It therefore follows that

$$
M_{2 k, \epsilon}=\frac{k !(-2 \epsilon \beta)_{2 k}}{(-\epsilon \beta)_{k}} .
$$

We furthermore have, also from 49,

$$
M_{2 k+1, \epsilon}=(2 k+1-2 \epsilon \beta) M_{2 k, \epsilon}=\frac{k !}{2} \frac{(-2 \epsilon \beta)_{2 k+2}}{(-\epsilon \beta)_{k+1}} .
$$




\section{The $n=2$ case with Klein deformed oscillators and $D(2,1 ; \alpha)$ spectrum-generating superalgebras}

The $n=2$ case corresponds to the $4 \times 4$ matrix oscillator. With respect to the $n=1$ case, the following features are encountered for the Klein deformation:

i) in the presence of a non-vanishing Klein deformation the $\operatorname{ssp}(4 \mid 2)$ spectrum-generating superalgebra of the undeformed case is deformed into a $D(2,1 ; \alpha)$ spectrum-generating superalgebra;

ii) all eigenstates of the model are doubly degenerate;

iii) the $D(2,1 ; \alpha)$ superalgebra is redundant to determine the spectrum of the theory since the ray vectors of the Hilbert space are determined by a $\operatorname{ssp}(2 \mid 2)$ subalgebra. It provides, nevertheless, a further information due to the fact that $\alpha$ is related with both the Klein deformation parameter and the vacuum energy of the model.

The operators are explicitely constructed in terms of the $\gamma_{J}(J=1,2, \ldots, 5)$ gamma matrices which can be introduced as follows

$$
\gamma_{1}=\sigma_{2} \otimes \sigma_{1}, \quad \gamma_{2}=\sigma_{2} \otimes \sigma_{2}, \quad \gamma_{3}=\sigma_{2} \otimes \sigma_{3}, \quad \gamma_{4}=\sigma_{1} \otimes \mathbb{I}_{2}, \quad \gamma_{5}=\sigma_{3} \otimes \mathbb{I}_{2} .
$$

The three $\sigma_{i}$ 's are the Pauli matrices introduced in (33). The block-diagonal matrix $\gamma_{5}=$ $\gamma_{1} \gamma_{2} \gamma_{3} \gamma_{4}$ is the fermion parity operator.

The eight Hermitian odd operators are $Q_{I}, \widetilde{Q}_{I}(I=1,2,3,4)$. The even Hermitian operators are $H, D, K$, closing the $s l(2)$ subalgebra, and $S_{i}, W_{i j}=-W_{j i}(i, j=1,2,3)$, closing the $R$ symmetry subalgebra. They are given by

$$
\begin{aligned}
Q_{I} & =\frac{i}{\sqrt{2}} \gamma_{I} \cdot\left(\partial_{x}+\frac{\beta \gamma_{5}}{x}\right), \\
\widetilde{Q}_{I} & =\frac{1}{\sqrt{2}} \gamma_{I} \cdot x, \\
H & =\frac{1}{2}\left(-\partial_{x}^{2}+\frac{\beta^{2}+\beta \gamma_{5}}{x^{2}}\right) \cdot \mathbb{I}_{4}, \\
D & =-\frac{i}{2}\left(x \partial_{x}+\frac{1}{2}\right) \cdot \mathbb{I}_{4}, \\
K & =\frac{1}{2} x^{2} \cdot \mathbb{I}_{4}, \\
S_{i} & =\frac{i}{2} \gamma_{4} \gamma_{i}\left(1-2 \beta \gamma_{5}\right), \\
W_{i j} & =\frac{i}{2} \gamma_{i} \gamma_{j}\left(1-2 \beta \gamma_{5}\right),
\end{aligned}
$$

where $\beta$ is the real deformation parameter.

Their non-vanishing (anti)commutators realize, see formula 103$)$, the $D(2,1 ; \alpha)$ superalgebra with the identification

$$
\alpha=\beta-\frac{1}{2}
$$

By repeating the analysis of the $n=1$ case one finds that the ray vectors of the Hilbert space of the model are determined by the $\operatorname{osp}(2 \mid 2) \subset D(2,1 ; \alpha)$ subalgebra. Different choices allow 
to pick up the $\operatorname{ssp}(2 \mid 2)$ spectrum-generating superalgebra. We can, e.g., select the operators to be given by $H, D, K, Q_{1}, Q_{3}, \widetilde{Q}_{1}, \widetilde{Q}_{3}, W_{13}$, where the latter operator is the $u(1) R$-symmetry of $\operatorname{osp}(2 \mid 2)$. An alternative choice consists of the set of operators $H, D, K, Q_{2}, Q_{4}, \widetilde{Q}_{2}, \widetilde{Q}_{4}, S_{2}$.

The four pairs of creation/annihilation operators are introduced, as usual, through the positions $a_{I}=Q_{I}+i \widetilde{Q}_{I}, a_{I}^{\dagger}=Q_{I}-i \widetilde{Q}_{I}$. The Klein-deformed commutators now read

$$
\left[a_{I}, a_{I}^{\dagger}\right]=\mathbb{I}_{4}-2 \beta \gamma_{5},
$$

while the $\beta$-deformed oscillator $H_{o s c}(\beta)$ is given by

$$
H_{\text {osc }}(\beta)=\frac{1}{2}\left\{a_{I}, a_{I}^{\dagger}\right\}=H+K=\frac{1}{2}\left(-\partial_{x}^{2}+x^{2}+\frac{\beta^{2}+\beta \gamma_{5}}{x^{2}}\right) \cdot \mathbb{I}_{4} .
$$

The commutators

$$
\left[H_{o s c}(\beta), a_{I}\right]=-a_{I}, \quad\left[H_{o s c}(\beta), a_{I}^{\dagger}\right]=a_{I}^{\dagger}
$$

are satisfied.

Four lowest weight vectors $|l w v\rangle$ are determined by the condition $a_{I}|l w v\rangle=0$ for $I=1,2,3,4$.

The creation operators $a_{I}^{\dagger}$ close the "soft" supersymmetry algebra (see [22])

$$
\left\{a_{I}^{\dagger}, a_{J}^{\dagger}\right\}=\delta_{I J} Z, \quad I, J=1,2,3,4, \quad\left[Z, a_{I}^{\dagger}\right]=0,
$$

where

$$
Z=2 H-2 K+4 i D
$$

is a ladder operator.

The special points $\alpha=0,-1\left(\beta= \pm \frac{1}{2}\right)$ correspond (see the comment in Appendix B) to the spectrum-generating superalgebra

$$
A(1,1) \oplus s u(2) \quad(\text { at } \quad \alpha=0,-1) .
$$

The selection of the Hilbert space follows the construction for the $n=1$ case. We have that

i) in the range $\beta \leq-\frac{1}{2}$ the admissible Hilbert space corresponds to a direct sum of the two bosonic lowest weight representations. For $\beta<-\frac{1}{2}$ this construction applies to the $D(2,1 ; \alpha)$ superalgebras with $\alpha$ belonging to the fundamental domains $F D_{1}$ and $F D_{2}$ given in 102 ;

ii) in the range $\beta \geq \frac{1}{2}$ the admissible Hilbert space corresponds to a direct sum of the two fermionic lowest weight representations. For $\beta>\frac{1}{2}$ this construction applies to $\alpha$ belonging to the fundamental domains $F D_{5}$ and $F D_{6}$ given in $(102)$;

iii) in the intermediate range $-\frac{1}{2}<\beta<\frac{1}{2}$ one can select the Hilbert space as given by the direct sum of the four (two bosonic and two fermionic) lowest weight representations. This case applies to $\alpha$ belonging to the fundamental domains $F D_{3}$ and $F D_{4}$ of formula $(102)$.

We now focus on the third case. The four normalized lowest weight vectors $|0\rangle_{\epsilon, \rho}, \epsilon, \rho= \pm 1$, are

$$
\begin{aligned}
|0\rangle_{1,1}=N_{1}\left(\begin{array}{c}
x^{-\beta} e^{-\frac{1}{2} x^{2}} \\
0 \\
0 \\
0
\end{array}\right), \quad|0\rangle_{1,-1}=N_{1}\left(\begin{array}{c}
0 \\
x^{-\beta} e^{-\frac{1}{2} x^{2}} \\
0 \\
0
\end{array}\right), \\
|0\rangle_{-1,1}=N_{-1}\left(\begin{array}{c}
0 \\
0 \\
x^{\beta} e^{-\frac{1}{2} x^{2}} \\
0
\end{array}\right), \quad|0\rangle_{-1,-1}=N_{-1}\left(\begin{array}{c}
0 \\
0 \\
0 \\
x^{\beta} e^{-\frac{1}{2} x^{2}}
\end{array}\right) .
\end{aligned}
$$


The states $|0\rangle_{1, \rho}\left(|0\rangle_{-1, \rho}\right)$ are bosonic (fermionic). The normalization factors $N_{ \pm 1}$ have been introduced in (47). The degeneracy of the bosonic (fermionic) energy eigenstates is removed by the eigenvalues of, let's say, the $S_{2}$ operator $\left(\left[S_{2}, \gamma_{5}\right]=\left[S_{2}, H_{o s c}(\beta)\right]=0\right)$.

By taking into account the $D(2,1 ; \alpha)$ redundancy, the Hilbert space is spanned by the following ray vectors which correspond to energy eigenstates

$$
\left(a_{1}^{\dagger}\right)^{n}\left(a_{3}^{\dagger}\right)^{m}|0\rangle_{\epsilon, \rho}=|\overline{n, m}\rangle_{\epsilon, \rho}, \quad n, m \in \mathbb{N}_{0} .
$$

Their corresponding energy eigenvalues are

$$
E_{n, m ; \epsilon, \rho}=\frac{1}{2}-\epsilon \beta+n+m
$$

The orthonormalized eigenvectors $|n, m\rangle_{\epsilon, \rho}$ are determined by applying the same techniques as in the $n=1$ case.

A similarity transformation, analogous to (41), is induced by the operator $\gamma_{4}$. Let $g$ denote an operator of (58). The similarity transformation is defined by

$$
g \mapsto g^{\prime}=\gamma_{4} g \gamma_{4}
$$

In particular

$$
H_{o s c}^{\prime}(\beta)=H_{o s c}(-\beta)
$$

Without loss of generality we can restrict $\beta$ to the non-negative axis $\beta \geq 0$. For the third choice of the Hamiltonian the range $0<\beta<\frac{1}{2}$ corresponds to the 102 fundamental domain $F D_{4}$ $\left(-\frac{1}{2}<\alpha<0\right)$ for $\alpha$. In this interval the lowest weight vectors $|0\rangle_{1, \pm 1}$ are the two degenerate bosonic vacua of the theory. The corresponding vacuum energy, expressed in terms of $\alpha$, is

$$
E_{v a c}=-\alpha .
$$

Even if $D(2,1 ; \alpha)$ is redundant as a spectrum-generating superalgebra, it encodes an important dynamical information of the theory.

We point out, as a final remark, that since $\alpha$ belongs to a fundamental domain, all inequivalent (for $\alpha$ real) $D(2,1 ; \alpha)$ superalgebras are spectrum-generating superalgebras of an associated dynamical system. Stating otherwise, there is no gap in the $\alpha$-induced spectrum generating superalgebras.

\section{The $n=2$ case with non-Klein deformed oscillators and $\operatorname{sep}(2 \mid 2)$ spectrum-generating superalgebra}

The next construction presents a non-Klein deformation of the $4 \times 4$ matrix oscillator. For this deformation the $\operatorname{sp}(4 \mid 2)$ spectrum generating superalgebra of the undeformed case is broken to a $\operatorname{osp}(2 \mid 2)$ spectrum-generating superalgebra.

In this construction the block-antidiagonal, Hermitian, constant matrices $M_{I}$ entering the (21) deformed supersymmetry operators are different from the ones expressed by (26). The $M_{I}$ 's are given by a linear combination $M_{I}=\nu \widetilde{\gamma}_{I}+i b \gamma_{I} \gamma_{5}$, where $\widetilde{\gamma}_{I}$ denotes, up to a sign, one of the gamma matrices (different from $\gamma_{I}$ and $\gamma_{5}$ ) entering (57). The requirement that the constraints (22 23 24 24 have to be satisfied implies that at most two deformed supersymmetry operators can be constructed, so that $I=1,2$. The requirement that $V_{I}=\frac{1}{2}\left(M_{I}^{2}-i \gamma_{I} M_{I}\right)$ is a diagonal matrix 
and, furthermore, $V_{1}=V_{2}$, implies that $b$ has to be set to the value $b=\frac{1}{2}$, while $\nu$ is an arbitrary real number.

It is easily proven that, without loss of generality (the other solutions being recovered from similarity transformations), an explicit expression of $Q_{1}, Q_{2}$ is given by

$$
\begin{aligned}
Q_{1} & =\frac{i}{\sqrt{2}}\left(\gamma_{1} \partial_{x}-i \frac{M_{1}}{x}\right), & \text { with } & M_{1} & =\nu \gamma_{2}+\frac{i}{2} \gamma_{1} \gamma_{5}, \\
Q_{2} & =\frac{i}{\sqrt{2}}\left(\gamma_{3} \partial_{x}-i \frac{M_{2}}{x}\right), & \text { with } & M_{2} & =-\nu \gamma_{4}+\frac{i}{2} \gamma_{3} \gamma_{5} .
\end{aligned}
$$

The $\gamma_{I}$ matrices were introduced in (57).

Besides $Q_{1}, Q_{2}$, the remaining operators entering the $\operatorname{ssp}(2 \mid 2)$ superalgebra are $H, K, D, J, \widetilde{Q}_{1}, \widetilde{Q}_{2}$. We have, in particular,

$$
H=-\frac{1}{2} \partial_{x}^{2} \cdot \mathbb{I}_{4}+\frac{1}{x^{2}} V
$$

where

$$
V=\frac{1}{8} \operatorname{diag}\left(4 \nu^{2}+8 \nu+3,4 \nu^{2}-8 \nu+3,4 \nu^{2}-1,4 \nu^{2}-1\right)
$$

and

$$
J=-i e_{34}+i e_{43}
$$

( $e_{i j}$ denotes the matrix with entry 1 at the $i$-th row and $j$-th column and 0 otherwise).

The operators $D, K, \widetilde{Q}_{1}, \widetilde{Q}_{2}$ are unaffected by the $M_{I}$ 's deformations. Within our conventions we have

$$
D=-\frac{i}{2}\left(x \partial_{x}+\frac{1}{2}\right) \cdot \mathbb{I}_{4}, \quad K=\frac{1}{2} x^{2} \cdot \mathbb{I}_{4}, \quad \widetilde{Q}_{1}=\frac{1}{\sqrt{2}} x \gamma_{1}, \quad \widetilde{Q}_{2}=\frac{1}{\sqrt{2}} x \gamma_{3}
$$

The non-vanishing (anti)commutators coincide (for the new identification of the corresponding $o s p(2 \mid 2)$ operators) with the ones given in (98).

Two $a_{I}, a_{I}^{\dagger}(I=1,2)$ pairs of deformed Heisenberg oscillators are introduced through

$$
a_{I}=Q_{I}+i \widetilde{Q}_{I}, \quad a_{I}^{\dagger}=Q_{I}-i \widetilde{Q}_{I} .
$$

They define the deformed Heisenberg algebras

$$
\left[a_{I}, a_{I}^{\dagger}\right]=\mathbb{I}_{4}+G_{I},
$$

where

$$
G_{1}=\operatorname{diag}(-1-2 \nu,-1+2 \nu, 1-2 \nu, 1+2 \nu), \quad G_{2}=\operatorname{diag}(-1-2 \nu,-1+2 \nu, 1+2 \nu, 1-2 \nu) .
$$

Since $G_{I}^{2}$ is not proportional to $\mathbb{I}_{4}$, these Heisenberg deformations are not of Klein type. One should also note that $G_{1} \neq G_{2}$.

The deformed oscillator $H_{o s c}$ is

$$
H_{\text {osc }}=H+K=\frac{1}{2}\left\{a_{1}, a_{1}^{\dagger}\right\}=\frac{1}{2}\left\{a_{2}, a_{2}^{\dagger}\right\}=\frac{1}{2}\left(-\partial_{x}^{2}+x^{2}\right) \cdot \mathbb{I}_{4}+\frac{1}{x^{2}} V .
$$


It follows, by taking into account the vanishing anticommutators

$$
\left\{G_{I}, a_{I}\right\}=\left\{G_{I}, a_{I}^{\dagger}\right\}=0
$$

that $a_{I}\left(a_{I}^{\dagger}\right)$ are annihilation (creation) operators satisfying

$$
\left[H_{o s c}, a_{I}\right]=-a_{I}, \quad\left[H_{o s c}, a_{I}^{\dagger}\right]=a_{I}^{\dagger}
$$

The bosonic (fermionic) states are the eigenfunctions of $\gamma_{5}$ with eigenvalue $+1(-1)$.

We are now in the position to introduce the lowest weight representations. A lowest weight vector $|l w v\rangle$ satisfies the condition

$$
a_{I}|l w v\rangle=0, \quad \text { for } \quad I=1,2 .
$$

Two (both bosonic) lowest weight vectors are found. They are given by $\Psi_{1,2}(x)$, where

$$
\Psi_{1}(x)=\left(\begin{array}{c}
x^{-\left(\frac{1}{2}+\nu\right)} e^{-\frac{1}{2} x^{2}} \\
0 \\
0 \\
0
\end{array}\right), \quad \Psi_{2}(x)=\left(\begin{array}{c}
0 \\
x^{\left(\nu-\frac{1}{2}\right)} e^{-\frac{1}{2} x^{2}} \\
0 \\
0
\end{array}\right) .
$$

The fermionic states $\Psi_{3}(x)=a_{1}^{\dagger} \Psi_{1}(x), \Psi_{4}(x)=a_{1}^{\dagger} \Psi_{2}(x), \Psi_{5}(x)=a_{2}^{\dagger} \Psi_{1}(x), \Psi_{6}(x)=a_{2}^{\dagger} \Psi_{2}(x)$ satisfy the conditions $a_{2} \Psi_{3}(x)=a_{2} \Psi_{4}(x)=a_{1} \Psi_{5}(x)=a_{1} \Psi_{6}(x)=0$. They are, nevertheless, excited states belonging to the lowest weight representations induced by $\Psi_{1,2}(x)$.

As recalled in Appendix $\mathbf{C}$, a wavefunction of the form $x^{\beta} e^{-\frac{1}{2} x^{2}}$ is normalized provided that $\beta>-\frac{1}{2}$. It follows that a normalized lowest weight vector is encountered, provided that

$$
\nu \neq 0 \text {. }
$$

In this range the Hilbert space is given by a single lowest weight representation. For $\nu<0$ the normalizable lowest weight state is $\Psi_{1}(x)$; for $\nu>0$ the normalizable lowest weight state is $\Psi_{2}(x)$. The vacuum energy $E_{v a c}$, in the admissible $\nu \neq 0$ range, is

$$
E_{v a c}=-\frac{1}{2}+|\nu| \text {. }
$$

The spectrum of the theory is given by

$$
E_{n}=-\frac{1}{2}+|\nu|+n, \quad n \in \mathbb{N}_{0} .
$$

With the exception of the single vacuum state, all excited states for $n \geq 1$ are doubly degenerate. This follows from the $\mathcal{N}=2$ soft supersymmetry algebra satisfied by the two creation operators $a_{I}^{\dagger}$, given by

$$
\left\{a_{I}^{\dagger}, a_{J}^{\dagger}\right\}=\delta_{I J} Z, \quad\left[Z, a_{I}^{\dagger}\right]=0
$$

with

$$
Z=2 H-2 K+4 i D .
$$

Therefore, the spectrum corresponds to the semi-infinite tower of $(1,2,2,2, \ldots)$ states. 
Let us consider the $\nu>0$ case. In this case the normalized vacuum state $|0\rangle$ is

$$
|0\rangle=N \Psi_{2}(x), \quad \text { with } \quad N=\frac{1}{\sqrt{\Gamma(\nu)}}
$$

The $n$ excited states are spanned by the vectors $\left(a_{1}^{\dagger}\right)^{n_{1}}\left(a_{2}^{\dagger}\right)^{n_{2}}|0\rangle$, where $n=n_{1}+n_{2}$. At given $n>0$, due to the (88) relation, only two of the associated ray vectors are distinct. They can be chosen to be expressed through

$$
|\overline{n, 0}\rangle=\left(a_{1}^{\dagger}\right)^{n}|0\rangle, \quad|\overline{n-1,1}\rangle=\left(a_{1}^{\dagger}\right)^{n-1} a_{2}^{\dagger}|0\rangle .
$$

By applying the method discussed in Section 3 we can compute the orthonormal states for $\nu>0$. We report here just the final results. The orthonormal states are

$$
|n, 0\rangle=N_{n, 0}|\overline{n, 0}\rangle, \quad|n-1,1\rangle=N_{n-1,1}|\overline{n-1,1}\rangle
$$

where

$$
\begin{aligned}
N_{n, 0} & =\left(2^{n}\left\lfloor\frac{n}{2}\right\rfloor !(\nu)_{\left\lceil\frac{n}{2}\right\rceil}\right)^{-1 / 2}, \\
N_{2 m-1,1} & =\frac{1}{\sqrt{2^{2 m}(m-1) !(\nu)_{m+1}}}, \quad N_{2 m, 1}=\frac{1}{\sqrt{2^{2 m+1} m !(\nu)_{m+1}}} .
\end{aligned}
$$

In the above equations $(\nu)_{m}$ denotes the Pochhammer symbol, while $\lfloor x\rfloor$ and $\lceil x\rceil$ are, respectively, the floor and ceiling functions.

\section{Comments on the general $n$ case}

In Section 3 we presented the conditions to be satisfied in order to have a spectrum-generating superalgebra for the inverse-square potential deformed matrix oscillators. A scale-invariant supersymmetric quantum mechanics is implied by fulfilling the conditions $(22)$ and (23). The existence of a spectrum-generating superconformal algebra is further implied by satisfying (24), plus the requirement for the fermionic generators to belong to a representation multiplet of the $R$-symmetry generators (25). We presented the most general solutions (up to similarity transformations) for $n=1$ (in Section 4) and $n=2$ (in Sections $\mathbf{5}$ and $\mathbf{6}$ for deformations of, respectively, Klein type and non-Klein type).

It is beyond the scope of the present paper to investigate the most general class of solutions for $n \geq 3$. This will be left to future works. It is worth, nevertheless, to introduce the present state of the art and discuss some general features which can be noted. Beyond $n=2$, a nontrivial solution was found in [24] for $n=4$ (the associated spectrum-generating superalgebra being $F(4)$, with $\mathcal{N}=8$ supersymmetries, see Appendix $\mathbf{A})$.

So far this is the only known non-trivial solution for $n \geq 3$. Its construction was made possible by the large symmetry of the model, reflected by the so-called "octonionic covariance" which, essentially, derives from the construction of its gamma matrices in terms of the octonionic structure constants. The model is unique (up to similarity transformations) and corresponds, even if not explicitly stated in [24], to a deformation of non-Klein type. The results of [24] rule out $n=4$ non-trivial octonionic covariant deformations based on the $\mathcal{N}=8$ superconformal algebra $\operatorname{osp}(8 \mid 2)$ and on the $\mathcal{N}=7$ exceptional superconformal algebra $G(3)$.

The following picture emerges: 
i) at $n=1$ the deformation is of Klein type and depends on a real continuous parameter $\beta$. In the $\beta \rightarrow 0$ limit the undeformed oscillator is recovered. The spectrum-generating superalgebra $\operatorname{osp}(2 \mid 2)$ is recovered for both undeformed and deformed oscillators;

ii) for $n=2$ two new features appear. The deformation of Klein type, which depends on a continuous parameter $\alpha$, is such that its spectrum-generating superalgebra is deformed, since the $\operatorname{osp}(4 \mid 2)$ spectrum-generating superalgebra of the undeformed oscillator (recovered in the $\alpha \rightarrow 0$ limit) is replaced by $D(2,1 ; \alpha)$. The second new feature is the appearance of the non-Klein deformation which depends on a continuous parameter $\nu \neq 0$. Contrary to the Klein type deformation, the non-Klein deformation is not connected with the undeformed oscillator;

iii) at $n=4$ the non-Klein deformation possesses the spectrum-generating superalgebra $F(4)$ and is point-like. It corresponds to an isolated point of the inverse-square potential coupling constants entering the diagonal matrix Hamiltonian. The deformation is obviously not connected with the $\operatorname{ssp}(8 \mid 2)$ undeformed oscillator.

\section{Conclusions}

The systematic construction of inverse-square potential deformed matrix oscillators with superconformal spectrum-generating superalgebras for larger $(n \geq 3)$ matrices is left for future works (the only case which is known, the [24] construction for $n=4$ and $F(4)$ superalgebra, is made possible by the simplifications due to its huge symmetry). In a forthcoming work we will present the results for $d$-dimensional, with $d \geq 2$, deformed matrix oscillators. Another promising future line of research consists in addressing the multi-particle case. It requires extending at the quantum level the construction which is done (see, eg., the recent [27] paper) for multi-particle classical superconformal world-line models.

We conclude with two more comments. The first one is the recognition that, since in a certain range of the deformation parameter the Hilbert space can be taken as a direct sum of lowest weight representations of its spectrum-generating superalgebra, therefore the superalgebra does not contain all information about the spectrum of the theory (not every higher energy excited state is connected to a given lower energy state via superalgebra ladder operators). This offers the tantalizing possibility that extra algebraic structures, possibly infinite-dimensional, could be responsible for that and used to generate the whole spectrum of the theory.

The final comment concerns the possible interesting applications of these models to higherspin theories (as recognized in 23]), in a implementation of the AdS/CFT holography. This is based on the property that Klein-deformed oscillators with $\operatorname{osp}(2 \mid 2)$ spectrum-generating superalgebra provide a realization of the Vasiliev's higher spin superalgebra introduced in [5]. Recently, the relevance of non-Klein deformed oscillators to higher spin theories was pointed out (see e.g. [28] and references therein).

\section{Appendix A: the $1 D$ finite superconformal Lie algebras}

The set of the one-dimensional finite superconformal Lie algebras is a subclass of the finite simple Lie superalgebras entering the Kac's classification [29] (see also the classification in [30] and, for exceptional superalgebras, 31, as well as the [32] review) and satisfying the following additional properties [33]. Any such Lie superalgebra $\mathcal{G}$ over the field $\mathbb{C}$ admits a 5 -grading 
decomposition

$$
\mathcal{G}=\mathcal{G}_{-1} \oplus \mathcal{G}_{-\frac{1}{2}} \oplus \mathcal{G}_{0} \oplus \mathcal{G}_{\frac{1}{2}} \oplus \mathcal{G}_{1}
$$

The (anti)commutators (compactly denoted as "[.,..\}") satisfy the condition

$$
\left[\mathcal{G}_{i}, \mathcal{G}_{j}\right\} \subset \mathcal{G}_{i+j}
$$

The even sector $\mathcal{G}_{\text {even }}=\mathcal{G}_{-1} \oplus \mathcal{G}_{0} \oplus \mathcal{G}_{1}$ is isomorphic to the direct sum of the Lie algebras $\operatorname{sl}(2) \oplus R$, where the subalgebra $R$ is known as $R$-symmetry.

The odd sector $\mathcal{G}_{\text {odd }}=\mathcal{G}_{-\frac{1}{2}} \oplus \mathcal{G}_{\frac{1}{2}}$ is spanned by $2 \mathcal{N}$ generators. Accordingly, each finite superconformal Lie algebra $\mathcal{G}$ is labeled by its associated positive integer $\mathcal{N}$.

The positive sector $\mathcal{G}_{>0}$ is isomorphic to the algebra of the $\mathcal{N}$-extended supersymmetric quantum mechanics [34, 35] defined by the (anti)commutators

$$
\left\{Q_{I}, Q_{J}\right\}=2 \delta_{I J} H, \quad\left[H, Q_{I}\right]=0, \quad \text { for } \quad I, J=1, \ldots, \mathcal{N} .
$$

The generator $H$ is the positive root of the $s l(2)$ subalgebra. The $s l(2)$ Cartan and negative root generators are denoted as $D, K$, respectively. The negative sector $\mathcal{G}_{<0}$ satisfies the subalgebra

$$
\left\{\widetilde{Q}_{I}, \widetilde{Q}_{J}\right\}=2 \delta_{I J} K, \quad\left[K, \widetilde{Q}_{I}\right]=0, \quad \text { for } \quad I, J=1, \ldots, \mathcal{N} .
$$

The sector $\mathcal{G}_{1}\left(\mathcal{G}_{-1}\right)$ is spanned by $H(K)$; the odd sector $\mathcal{G}_{\frac{1}{2}}\left(\mathcal{G}_{-\frac{1}{2}}\right)$ is spanned by the $Q_{I}\left(\widetilde{Q}_{I}\right)$ generators; finally, the $\mathcal{G}_{0}$ sector is $\mathcal{G}_{0}=D \mathbb{C} \oplus R$.

In this paper on spectrum-generating superalgebras of the matrix inverse-square potential models several examples of one-dimensional conformal Lie superalgebras appear. In particular we mentioned superalgebras belonging to the classical series, such as $D(\mathcal{N}, 1) \sim \operatorname{osp}(2 \mathcal{N} \mid 2)$ (defined for any positive $\mathcal{N}$ and with $s o(2 \mathcal{N})$ as associated R-symmetry) and $B(n, 1) \sim o s p(2 n+$ $1 \mid 2)$ (such that $\mathcal{N}=2 n+1$ and with $s o(2 n+1)$ as R-symmetry), as well as the exceptional superalgebras $D(2,1 ; \alpha)$ (superconformal for $\mathcal{N}=4$ and discussed in Appendix $\mathbf{B}$ ), $G(3)$ and $F(4)$. The latter two exceptional superalgebras are superconformal for, respectively, $\mathcal{N}=7$ with $g_{2}$ as R-symmetry and $\mathcal{N}=8$ with $s o(7)$ as R-symmetry.

The list of one-dimensional superconformal Lie algebras further includes $A(n, 1), D(2, n)$ (see [32 for their definition). The complete list of one-dimensional superconformal Lie algebras with $\mathcal{N} \leq 8$ is presented in 33 .

We present here for convenience the non-vanishing (anti)commutators of the $\operatorname{ssp}(2 \mid 2)$ superalgebra generators given by the (34) operators, with $I, J=1,2$. We have

$$
\begin{array}{ccc}
{[D, K]=-i K,} & {[D, H]=i H,} & {[H, K]=-2 i D,} \\
\left\{Q_{I}, Q_{J}\right\}=2 \delta_{I J} H, & \left\{\widetilde{Q}_{I}, \widetilde{Q}_{J}\right\}=2 \delta_{I J} K, & \left\{Q_{I}, \widetilde{Q}_{J}\right\}=-2 \delta_{I J} D+\epsilon_{I J} J, \\
{\left[D, Q_{I}\right]=\frac{i}{2} Q_{I},} & {\left[D, \widetilde{Q}_{I}\right]=-\frac{i}{2} \widetilde{Q}_{I},} \\
{\left[Q_{I}, K\right]=i \widetilde{Q}_{I},} & {\left[\widetilde{Q}_{I}, H\right]=-i Q_{I},} \\
{\left[J, Q_{I}\right]=-i \epsilon_{I J} Q_{J},} & {\left[J \widetilde{Q}_{I}\right]=-i \epsilon_{I J} \widetilde{Q}_{J} .}
\end{array}
$$

In the above relations $\epsilon_{12}=-\epsilon_{21}=1$ is the totally antisymmetric tensor.

\section{Appendix B: basic properties of the $D(2,1 ; \alpha)$ superalgebras}


The exceptional superalgebras $D(2,1 ; \alpha)$ are parametrized, see [32], by $\alpha \in \mathbb{C} \backslash\{0,-1\}$, with $\alpha$ entering the structure constants. As recalled in Appendix $\mathbf{A}$, they are $\mathcal{N}=4$ superconformal Lie algebras. Their even sector $\mathcal{G}_{\text {even }}$ is the direct sum of three $\operatorname{sl}(2)$ subalgebras, so that

$$
\mathcal{G}_{\text {even }}=\operatorname{sl}(2) \oplus \operatorname{sl}(2) \oplus \operatorname{sl}(2) .
$$

The three $s l(2)$ subalgebras can be interchanged. As a result, the $S_{3}$ permutation group of three elements acts on $\alpha$; two generators of $S_{3}$ are expressed as the transformations $\alpha \mapsto \frac{1}{\alpha}$, $\alpha \mapsto-(1+\alpha)$. An $S_{3}$-orbit is given by the following set of elements

$$
\left\{\alpha, \quad \frac{1}{\alpha}, \quad-(1+\alpha), \quad-\frac{1}{(1+\alpha)}, \quad-\frac{(1+\alpha)}{\alpha}, \quad-\frac{\alpha}{(1+\alpha)}\right\} .
$$

The superalgebras specified by $\alpha, \alpha^{\prime}$ belonging to the same $S_{3}$-orbit are isomorphic.

The special values

$$
\alpha=-2,-\frac{1}{2}, 1
$$

correspond to the superalgebra $D(2,1) \sim \operatorname{osp}(4 \mid 2)$ which belongs to the classical series of orthosymplectic superalgebras.

The structure constants can also be defined at the special values $\alpha=0,-1$. For these values, on the other hand, the superalgebra is no longer simple, being given by the direct sum $A(1,1) \oplus s l(2)$ (the generators of one of the three $s l(2)$ subalgebras decouple from the remaining generators). The simple superalgebra $A(1,1)$ is $\mathcal{N}=4$ superconformal.

The hermiticity property of the Hamiltonians of the matrix inverse-square potential quantum mechanics (both in presence or in absence of the oscillatorial term) requires $\alpha$ to be real. For $\alpha \in$ $\mathbb{R}$, the following six fundamental domains under the group of $S_{3}$ transformations are encountered [14]:

$$
\begin{array}{lrl}
F D_{1}: & -\infty<\alpha \leq-2, \\
F D_{2}: & -2 \leq \alpha<-1, \\
F D_{3}: & -1<\alpha \leq-\frac{1}{2}, \\
F D_{4}: & -\frac{1}{2} \leq \alpha<0, \\
F D_{5}: & 0<\alpha \leq 1, \\
F D_{6}: & 1 \leq \alpha<\infty .
\end{array}
$$

The operators given in formula $(58)$ produce the $D(2,1 ; \alpha)$ superalgebra; their non-vanishing 
(anti)commutators are given by

$$
\begin{aligned}
& {[D, K]=-i K,} \\
& {[D, H]=i H \text {, }} \\
& {[H, K]=-2 i D,} \\
& \left\{Q_{I}, Q_{J}\right\}=2 \delta_{I J} H, \\
& \left\{\widetilde{Q}_{I}, \widetilde{Q}_{I}\right\}=2 \delta_{I J} K, \\
& \left\{Q_{I}, \widetilde{Q}_{I}\right\}=-2 D, \\
& \left\{Q_{4}, \widetilde{Q}_{i}\right\}=S_{i}, \\
& \left\{\widetilde{Q}_{4}, Q_{i}\right\}=-S_{i}, \\
& \left\{Q_{i}, \widetilde{Q}_{j}\right\}=W_{i j} \text {, } \\
& {\left[D, Q_{I}\right]=\frac{i}{2} Q_{I},} \\
& {\left[D, \widetilde{Q}_{I}\right]=-\frac{i}{2} \widetilde{Q}_{I},} \\
& {\left[Q_{I}, K\right]=i \widetilde{Q}_{I},} \\
& {\left[\widetilde{Q}_{I}, H\right]=-i Q_{I},} \\
& {\left[Q_{4}, S_{i}\right]=i Q_{i},} \\
& {\left[\widetilde{Q}_{i}, S_{j}\right]=-i \delta_{i j} \widetilde{Q}_{4}+2 i \beta \epsilon_{i j k} \widetilde{Q}_{k},} \\
& {\left[Q_{i}, S_{j}\right]=-i \delta_{i j} Q_{4}+2 i \beta \epsilon_{i j k} Q_{k} \text {, }} \\
& {\left[\widetilde{Q}_{4}, W_{i j}\right]=-2 i \beta \epsilon_{i j k} \widetilde{Q}_{k} \text {, }} \\
& {\left[Q_{4}, W_{i j}\right]=-2 i \beta \epsilon_{i j k} Q_{k},} \\
& {\left[\widetilde{Q}_{i}, W_{j k}\right]=i\left(\delta_{i j} \widetilde{Q}_{k}-\delta_{i k} \widetilde{Q}_{j}\right)+2 i \beta \epsilon_{i j k} \widetilde{Q}_{4},} \\
& {\left[Q_{i}, W_{j k}\right]=i\left(\delta_{i j} Q_{k}-\delta_{i k} Q_{j}\right)+2 i \beta \epsilon_{i j k} Q_{4},} \\
& {\left[S_{i}, S_{j}\right]=-i W_{i j}+2 i \beta \epsilon_{i j k} S_{k},} \\
& {\left[S_{i}, W_{j k}\right]=i \delta_{i j}\left(S_{k}-\beta \epsilon_{k \ell m} W_{\ell m}\right)-i \delta_{i k}\left(S_{j}-\beta \epsilon_{j \ell m} W_{\ell m}\right),} \\
& {\left[W_{i j}, W_{k l}\right]=i\left(\delta_{i k} \widetilde{W}_{\ell j}-\delta_{i \ell} \widetilde{W}_{k j}+\delta_{j k} \widetilde{W}_{i \ell}-\delta_{j \ell} \widetilde{W}_{i k}\right),}
\end{aligned}
$$

where $\widetilde{W}_{i j}=W_{i j}-2 \beta \epsilon_{i j k} S_{k}$. The deformation parameter $\beta$ entering the 58 operators and the above (anti)commutators is related to $\alpha$ through the equation $\alpha=\beta-\frac{1}{2}$.

\section{Appendix C: selecting the Hilbert spaces of the models}

The selection of the viable Hilbert spaces of the matrix superconformal quantum mechanics (with or without the addition of the extra oscillatorial term) requires the preliminary knowledge of the selection of the Hilbert spaces for either the one-dimensional inverse-square potential model [3] defined by the Hamiltonian

$$
H_{\text {def }}=\frac{1}{2}\left(-\partial_{x}^{2}+\frac{g}{x^{2}}\right)
$$

or the de Alfaro-Fubini-Furlan model [4] defined by the Hamiltonian

$$
H_{D F F}=\frac{1}{2}\left(-\partial_{x}^{2}+\frac{g}{x^{2}}+x^{2}\right)
$$


An extensive analysis of the admissible choices of their Hilbert spaces for $g>0$ was given in [1, 2. We present here, in a slightly modified form suitable for our purposes, the results of [4, 1, 2] concerning the choice of the Hilbert spaces for the $H_{D F F}$ (105) Hamiltonian.

Following [36], the ground state wave function of $H_{D F F}$ has the form

$$
\Psi_{\beta}=x^{\beta} e^{-\frac{1}{2} x^{2}} .
$$

$\Psi_{\beta}(x)$ is an eigenfunction (not necessarily the groundstate) of $H_{D F F}$ provided that the relation

$$
g=\beta^{2}-\beta
$$

holds. Its associated energy eigenvalue $E_{\beta}$ is

$$
E_{\beta}=\frac{1}{2}+\beta .
$$

The two solutions of the (107) equation are $\beta_{ \pm}$, given by

$$
\beta_{ \pm}=\frac{1 \pm \sqrt{1+4 g}}{2} .
$$

The reality of $E_{\beta}$ requires $\beta$ to be real; therefore the coupling constant $g$ needs to be

$$
g \geq-\frac{1}{4}
$$

The wave function $\Psi_{\beta}(x)$ is square integrable in the real line provided that

$$
\int_{-\infty}^{+\infty} d x\left|\Psi_{\beta}(x)\right|^{2}=\int_{-\infty}^{+\infty} d x x^{2 \beta} e^{-x^{2}}=C_{\beta}<\infty .
$$

Taking into account the singularity at the origin for negative $\beta$, the above condition is satisfied for

$$
\beta>-\frac{1}{2}
$$

In the range

$$
\beta>0
$$

the wavefunction $\Psi_{\beta}(x)$ can be defined in the $x \geq 0$ non negative half line $\mathbb{R}^{+}$; it satisfies the Dirichlet boundary condition at the origin $\left(\Psi_{\beta}(0)=0\right)$.

In the range

$$
-\frac{1}{2}<\beta \leq 0
$$

the wavefunction $\Psi_{\beta}(x)$ is necessarily defined as $\mathcal{L}^{2}(\mathbb{R})$ square-integrable function on the real line.

The dynamical symmetry of the time-dependent Schrödinger equation with 104 or 105 as Hamiltonian is, see e.g. [23], $s l(2) \oplus u(1)$, so that the $s l(2)$ algebra is the spectrum generating algebra. The wavefunctions $\Psi_{\beta_{ \pm}}(x)$ are the lowest weight vectors of the $s l(2)$ lowest weight representations associated with $H_{D F F}$. All excited states obtained by applying the raising ladder operators to $\Psi_{\beta_{ \pm}}(x)$ belong, for $\beta_{ \pm}>0$, to the functions on the half line which satisfy the Dirichlet boundary condition and, for $-\frac{1}{2}<\beta_{ \pm} \leq 0$, to the $\mathcal{L}^{2}(\mathbb{R})$ square-integrable functions on the real line.

The Hilbert space of the model is either a single lowest weight representation of $\operatorname{sl}(2)$ or the direct sum of its two lowest weight representations.

In the admissible $g \geq-\frac{1}{4}$ interval of the coupling constant we have: 
i) at $g=-\frac{1}{4}, \beta_{+}=\beta_{-}=\frac{1}{2}$, so that there is a single lowest weight representation; its wavefunctions are defined on the half line and satisfy the Dirichlet boundary condition;

ii) in the range $-\frac{1}{4}<g<0, \beta_{ \pm}$are both positive. The Hilbert space is the direct sum of the two lowest weight representations. Its wavefunctions are defined on the half line and satisfy the Dirichlet boundary condition. $\Psi_{\beta_{-}}(x)$ is the ground state;

iii) at $g=0, H_{D F F}$ is the Hamiltonian of the ordinary one-dimensional oscillator. The two lowest weight representations correspond to wavefunctions which are respectively even (odd) under the $x \mapsto-x$ parity transformation. The gaussian $\Psi_{\beta_{-}}(x)$ is the ground state and the lowest state of the even parity eigenfunctions. The first excited state is given by $\Psi_{\beta_{+}}(x)$, the lowest weight vector of the odd-parity eigenfunctions;

iv) in the range $0<g<\frac{3}{4}, \beta_{+}$is positive while $\beta_{-}$is negative. Following [1, 2], two choices of Hilbert space can be made. Either the single lowest weight representation with $\Psi_{\beta_{+}}(x)$ as lowest weight vector (correponding to functions on the half line with Dirichlet boundary condition at the origin), or the direct sum of the two lowest weight representations corresponding to square integrable functions on the real line. In this latter case $\Psi_{\beta_{-}}(x)$ is the ground state;

v) for $g \geq \frac{3}{4}$, since $\beta_{-} \leq-\frac{1}{2}$, the wavefunction $\Psi_{\beta_{-}}(x)$ does not satisfy 111 and is not normalized. The Hilbert space is given by a single $s l(2)$ lowest weight representation with $\Psi_{\beta_{+}}(x)$ as lowest weight vector and ground state.

In all above cases the Hamiltonian $H_{D F F}$ is well defined as a self-adjoint operator acting on the corresponding Hilbert space. Its eigenvalues are discrete and bounded from below.

\section{Acknowledgments}

N. A. is supported by the grants-in-aid from JSPS (Contract No. 26400209). He thanks UFABC and CBPF for hospitality. I. E. C. acknowledges a CNPq Ph.D. grant. Z. K. and F. T. are grateful to the Osaka Prefecture University for hospitality. The work was supported by CNPq (PQ grant 308095/2017-0).

\section{References}

[1] H. Miyazaki and I. Tsutsui, Ann. Phys. 299 (2002) 78; arXiv:quant-ph/0202037.

[2] L. Fehér, I. Tsutsui and T. Fülöp, Nucl. Phys. B 715 (2005) 713; arXiv:math-ph/0412095. 3; arXiv:1112.0995[hep-th].

[3] F. Calogero, J. Math. Phys. 10 (1969) 2191.

[4] V. de Alfaro, S. Fubini and G. Furlan, Nuovo Cim. A 34 (1976) 569.

[5] M. A. Vasiliev, Int. J. Mod. Phys. A 6 (1991) 1115.

[6] M. S. Plyushchay, Ann. Phys. 245 (1996) 339; arXiv:hep-th/9601116.

[7] P. J. Olver, Applications of Lie Groups to Differential Equations - 2nd Edition, Springer, New York (1991). 
[8] R. Britto-Pacumio, J. Michelson, A. Strominger, and A. Volovich, Lectures on superconformal quantum mechanics and multi-black hole moduli spaces, in Progress in String Theory and M-Theory, NATO Science Series Vol. 564, Kluwer Acad. Press (2001) 235; arXiv:hepth/9911066.

[9] A. Sen, JHEP 0811 (2008) 075; arXiv:0805.0095[hep-th].

[10] C. Chamon, R. Jackiw, S-Y. Pi and L. Santos, Phys. Lett. B 701 (2011) 503; arXiv:1106.0726 hep-th].

[11] S. Fedoruk, E. Ivanov and O. Lechtenfeld, J. Phys. A 45 (2012) 173001; arXiv:1112.1947[hep-th].

[12] A. Pashnev and F. Toppan, J. Math. Phys. 42 (2001) 5257; arXiv:hep-th/0010135.

[13] Z. Kuznetsova, M. Rojas and F. Toppan, JHEP 0603 (2006) 098; arXiv:hep-th/0511274.

[14] Z. Kuznetsova and F. Toppan, J. Math. Phys. 53 (2012) 043513; arXiv:1112.0995[hep-th].

[15] S. Khodaee and F. Toppan, J. Math. Phys. 53 (2012) 103518; arXiv:1208.3612 hep-th].

[16] N. L. Holanda and F. Toppan, J. Math. Phys. 55 (2014) 061703; arXiv:1402.7298[hep-th].

[17] G. Papadopoulos, Class. Quant. Grav. 30 (2013) 075018; arXiv:1210.1719 hep-th].

[18] S. Fedoruk, E. Ivanov and O. Lechtenfeld, JHEP 0908 (2009) 081; arXiv:0905.4951[hep-th].

[19] S. Fedoruk, E. Ivanov and O. Lechtenfeld, JHEP 1004 (2010) 061703; arXiv:1402.7298[hepth].

[20] E. Ivanov and S. Sidorov, Class. Quantum Grav. 31 (2014) 075013; arXiv:1307.7690[hep-th].

[21] E. Ivanov, O. Lechtenfeld and S. Sidorov, JHEP 11 (2016) 031; arXiv:1609.00490[hep-th].

[22] I. E. Cunha, N. L. Holanda and F. Toppan, Phys. Rev. D 96 (2017) 065014; arXiv:1610.07205[hep-th].

[23] F. Toppan and M. Valenzuela, Adv. Math. Phys. (2018), ID 6263150; arXiv:1705.04004[hepth].

[24] N. Aizawa, Z. Kuznetsova and F. Toppan, J. Math. Phys. 59 (2018) 022101; arXiv:1711.02923 [math-ph].

[25] S. Okubo, J. Math. Phys. 32 (1991) 1657.

[26] H. L. Carrion, M. Rojas and F. Toppan, JHEP 0304 (2003) 040; arXiv:hep-th/0302113.

[27] S. Fedoruk and E. Ivanov, Multiparticle $\mathcal{N}=8$ mechanics with $F(4)$ superconformal symmetry, arXiv:1810.13366[hep-th].

[28] M. A. Vasiliev, From Coxeter Higher-Spin Theories to Strings and Tensor Models, arXiv:1804.06520[hep-th].

[29] V. G. Kac, Comm. Math. Phys. 53 (1977) 31.

[30] M. Scheunert, W. Nahm and V. Rittenberg, J. Math. Phys. 17 (1976) 1626. 
[31] M. Scheunert, W. Nahm and V. Rittenberg, J. Math. Phys. 17 (1976) 1640.

[32] L. Frappat, A. Sciarrino and P. Sorba, Dictionary on Lie algebras and superalgebras, Academic Press, London (2000); arXiv:hep-th/9607161.

[33] F. Toppan, Nankai Series in Pure App. Math. and Th. Phys. 11 (2013), 417; arXiv: 1302.3459 [math-ph].

[34] E. Witten, Nucl. Phys. B 188 (1981) 513.

[35] M. de Crombrugghe and V. Rittenberg, Ann. of Phys. 151 (1983) 99.

[36] M. A. Olshanetsky and A. M. Perelomov, Phys. Rep. 94 (1983) 313. 